\title{
G sonstam \\ Gait Seed and Sagittal Abdominal Diameter: The Results from the Handan Eye Study
}

\section{Juping Liu ( $\nabla$ tydljp@126.com )}

Tianjin Branch of National Clinical Research Center for Ocular Disease, Tianjin Medical University Eye Hospital

\section{Jie Hao}

Beijing Tongren Eye Center, Beijing Tongren Hospital, Capital Medical University, Beijing Institute of Ophthalmology

\section{Ye Zhang}

Beijing Tongren Eye Center, Beijing Tongren Hospital, Capital Medical University, Beijing Institute of Ophthalmology

\section{Kai Cao}

Beijing Tongren Eye Center, Beijing Tongren Hospital, Capital Medical University, Beijing Institute of Ophthalmology

\section{Xiaorong Li}

Tianjin Branch of National Clinical Research Center for Ocular Disease, Tianjin Medical University Eye Hospital

\section{Ningli Wang}

Beijing Tongren Eye Center, Beijing Tongren Hospital, Capital Medical University, Beijing Institute of Ophthalmology

\section{Research Article}

Keywords: gait speed, sagittal abdominal diameter, gender, age

Posted Date: October 26th, 2021

DOI: https://doi.org/10.21203/rs.3.rs-982216/v1

License: (c) (1) This work is licensed under a Creative Commons Attribution 4.0 International License. Read Full License 


\section{Abstract \\ Background}

The sagittal abdominal diameter (SAD) is more precise than body mass index (BMI) for predicting adverse events in elderly. While physical function and $\mathrm{BMI}$ is related, the relationship is uncertain. BMI and gait speed (GS) is related and have a U-shaped distribution. The objective was to examine the relationship between GS and SAD in men and women aged 50 years and older.

\section{Methods}

This was a cross-sectional analysis. Data from the Handan Eye Study (HES), a Chinese prospective longitudinal study with participants randomly selected from the Yongnian county. Usual GS was measured over a 4 meters-track. SAD was categorized by interquartile: $<18.0 \mathrm{~cm} ; 18.0-19.79 \mathrm{~cm} ; 19.8$ $21.89 \mathrm{~cm} ; \geq 21.9 \mathrm{~cm}$. Unadjusted and adjusted analyses of covariance were performed to estimate the gender-specific means (and 95\% $\mathrm{Cl}$ ) of GS (in $\mathrm{m} / \mathrm{s}$ ) according to SAD categories.

\section{Results}

The current analyses were performed in 2852 participants. Mean age was 56.16 years for women and 56.54 years for men. The unadjusted means of GS were 0.995 (95\% Cl 0.972-1.019) m/s in SAD Q1 participants, 0.991 (95\% Cl 0.968-1.014) m/s in SAD Q2, 0.986 (95\% Cl 0.964-1.007) m/s in SAD Q3 and 0.961 (95\% $\mathrm{Cl} 0.937-0.985) \mathrm{m} / \mathrm{s}$ in SAD Q4 individuals in women. The similar trend presented in men [Q1: 0.993 (95\% Cl 0.969-1.016) m/s; Q2: 0.980 (95\%Cl 0.956-1.004); Q3: 0.944 (95\% Cl 0.918-0.970); Q4: 0.948 $(95 \% \mathrm{Cl} 0.923-0.973)]$. After adjustment for age, the reported trends between $\mathrm{GS}$ and SAD in categories were largely confirmed in women, but not in men.

\section{Conclusions}

Age and gender should be considered when we explore the relationship between GS and SAD in elderly.

\section{Background}

Ageing and its associated social problems have turned into a common concern in many countries. Physical function decline is the main component of ageing and also is critical risk factor for adverse events of ageing such as mortality, fall, disability, hospitalization [1]. Gait speed (GS) is a generally utilized estimation to assess to evaluate the physical function. Besides, GS has been viewed as a good predictor for death, cognitive impairment, depression and Alzheimer's disease in elderly [2]. 
Obesity is a prevalent disease in industrialized countries and has shown to significantly predict negative outcome. Other than clinical comorbidity, it is associated with subclinical conditions such as inflammation and oxidative damage. Body mass index (BMI) is a typical index to explore and classify obesity and be perceived as a marker in a few conditions such as systemic diseases, well-being and frailty, etc. In recent years, lots of studies uncovered that sagittal abdominal diameter (SAD) was a better indicator of cardiovascular disease, hypertension and diabetes than $\mathrm{BMI}$ and waist circumference $[3,4]$. Previous studies have explored the relationship between BMI and GS, which demonstrated U-shaped relationship [5,6], but not all studies affirmed reliably. No study has yet explored this relationship between SAD and GS in 50 years and older in a Chinese rural area.

Since BMI and GS are related and have a U-shaped distribution, we hypothesize that SAD (a better predictor than BMI) may also be associated with GS. Therefore, the purpose of this study is to explore whether SAD and GS are associated or not and to indicate their correlation types among village-dwelling older adults aged 50 years and older.

\section{Methods}

\section{Data source and study population}

The Handan Eye Study (HES) is a population-based longitudinal study in Handan, Hebei Province, China. In 2006-2007, 6830 subjects of 7557 individuals (response rate:90.4\%) participated in the baseline study. In 2012-2013, 5394 subjects (85.3\%) of the survivors took part in the follow up study. GS test was performed in subjects aged 50 years or above. A detailed description of the methodology used for the HES can be found in previous publications [7, 8]. Beijing Tongren Hospital Ethics Committee (TREC200622) approved the HES according to the principles embodied in the Declaration of Helsinki and all participants provided written informed consent.

The data used in this study were collected from the HES follow-up study because SAD measurement was conducted only in the follow-up study. A comprehensive questionnaire, laboratory tests, an extensive eye examination procedure and an anthropomorphic procedure which included standing height, weight, SAD, waist circumference, hip circumference and blood pressure were undergone by trained nurses and examiners.

\section{Sagittal Abdominal Diameter}

SAD was measured using a portable sliding-beam caliper (Holtain Ltd, Crymych, UK). Participants rested on a lightly padded bed in a flexed position when the examiner marked the level of the iliac crests. The examiner lowered the caliper upper arm, lightly touching the abdomen. The SAD was recorded in duplicate to the nearest $0.1 \mathrm{~cm}$ [9]. 


\section{Gait Speed}

Participants performed the 4-meter gait speed (GS) test according to standardized instructions [10]. Participants were asked to walk a distance of 4 meters at their usual pace, starting from a still position. Start the timer with the first footfall after the 0-m line and stop the timer with the first footfall after the 4$\mathrm{m}$ line.GS was operationalized as the ratio between distance and time, and expressed in meters by seconds $(\mathrm{m} / \mathrm{s})$.

\section{Other Variables}

A detailed interviewer-administered questionnaire which included socio-demographics, self-reported chronic diseases and living behaviors such as smoking, drinking was used. Diabetes and hypertension were defined according to self-report from participants (previously diagnosed).

\section{Statistical analysis}

The data are presented as the means and standard deviations for continuous variables and as frequencies and percentages for categorical variables. All analyses were independently conducted for men and women to evaluate the gender differences for SAD. SAD was categorized by interquartile. Unadjusted and adjusted analyses of covariance were performed to assess the gender-specific means (and $95 \% \mathrm{Cl}$ ) of GS (in $\mathrm{m} / \mathrm{s}$ ) as indicated by SAD categories. After having provided results from the unadjusted analyses, age-adjusted models were performed. Spearman correlation coefficients were utilized to clarify the associations between SAD and GS. P-values $<0.05$ were considered statistically significant and $95 \%$ confidence intervals $(95 \% \mathrm{Cl})$ are provided. All analyses were performed using SPSS, version 24.0 (SPSS Inc., Chicago, IL, USA).

\section{Results}

Among the 5394 participants enrolled in the HES follow-up study, 3297 were aged 50 years or older. We choose the sample because SAD only performed in the HES follow-up study and GS test in elderly aged 50 years or above. The current analyses were performed in 2852 (86.5\%) participants,

after exclusion of 445 (13.5\%) individuals with missing data for the main variables of interest (GS or/and SAD).

Table 1 shows that the excluded 445 were older $(72.5 \pm 3.67$ versus $58.33 \pm 4.77)$, had a lower hip

circumference $(100.72 \pm 10.03 \mathrm{~cm}$ versus $101.88 \pm 9.83 \mathrm{~cm})$ and higher hypertension $(10.6 \%$ versus $8.4 \%)$. There were no significant differences in BMI, waist circumference, systolic blood pressure, diastolic blood pressure, SAD and the status of diabetes, smoking, alcohol drinking between the two groups. 
Table 2

Main characteristics of the study sample according to gender

\begin{tabular}{|llll|}
\hline & Women $(\mathrm{N}=1559)$ & Men $(\mathrm{N}=1293)$ & $P$ \\
\hline Age (years) & $56.16 \pm 4.72$ & $56.54 \pm 4.81$ & 0.039 \\
\hline Systolic BP $(\mathrm{mmHg})$ & $146.02 \pm 22.00$ & $145.98 \pm 21.76$ & 0.960 \\
\hline Diastolic BP $(\mathrm{mmHg})$ & $82.39 \pm 12.16$ & $82.57 \pm 11.91$ & 0.693 \\
\hline BMI $\left(\mathrm{kg} / \mathrm{m}^{2}\right)$ & $25.48 \pm 3.83$ & $25.61 \pm 3.97$ & 0.341 \\
\hline Waist circumference $(\mathrm{cm})$ & $89.10 \pm 11.50$ & $89.78 \pm 11.54$ & 0.124 \\
\hline Hip circumference $(\mathrm{cm})$ & $102.13 \pm 9.75$ & $101.59 \pm 9.94$ & 0.150 \\
\hline SAD $(\mathrm{cm})$ & $20.31 \pm 3.12$ & $19.85 \pm 3.06$ & 0.000 \\
\hline 4m gait speed $(\mathrm{m} / \mathrm{s})$ & $0.98 \pm 0.23$ & $0.97 \pm 0.23$ & 0.045 \\
\hline
\end{tabular}


Table 1

Comparison of subjects included and non-participated from GS data analysis

\begin{tabular}{|c|c|c|c|}
\hline & Included (N=2852) & Non-participates $(\mathrm{N}=445)$ & $\mathbf{p}$ \\
\hline Age (years) & $58.33 \pm 4.77$ & $72.50 \pm 3.67$ & 0.000 \\
\hline Female & $89.6 \%$ & $90.9 \%$ & 0.217 \\
\hline Systolic BP (mmHg) & $145.65 \pm 21.99$ & $144.16 \pm 26.51$ & 0.523 \\
\hline Diastolic BP (mmHg) & $81.99 \pm 12.10$ & $82.04 \pm 13.26$ & 0.956 \\
\hline $\mathrm{BMI}\left(\mathrm{kg} / \mathrm{m}^{2}\right)$ & $25.54 \pm 3.89$ & $25.60 \pm 3.90$ & 0.866 \\
\hline Waist circumference (cm) & $89.41 \pm 11.52$ & $89.46 \pm 11.34$ & 0.93 \\
\hline Hip circumference (cm) & $101.88 \pm 9.83$ & $100.72 \pm 10.03$ & 0.031 \\
\hline $\mathrm{SAD}(\mathrm{mm})$ & $201.00 \pm 30.96$ & $199.32 \pm 30.89$ & 0.301 \\
\hline \multicolumn{4}{|l|}{ Diabetes } \\
\hline Yes & $9.9 \%$ & $10.0 \%$ & \\
\hline No & $90.1 \%$ & $90.0 \%$ & 1.000 \\
\hline \multicolumn{4}{|l|}{ Hypertension } \\
\hline Yes & $8.4 \%$ & $10.6 \%$ & \\
\hline No & $91.6 \%$ & $89.4 \%$ & 0.041 \\
\hline \multicolumn{4}{|l|}{ Smoking } \\
\hline Yes & $10.2 \%$ & $8.1 \%$ & \\
\hline No & $89.8 \%$ & $91.9 \%$ & 0.099 \\
\hline \multicolumn{4}{|l|}{ Drinking } \\
\hline Yes & $9.6 \%$ & $9.9 \%$ & \\
\hline No & $90.4 \%$ & $90.1 \%$ & 0.93 \\
\hline
\end{tabular}

Table 2 shows the main characteristics according to gender. The mean age of the 2852 participants [1559 women (54.7\%) and 1293 men (45.3\%)] was 56.16 years for women and 56.54 years for men ( $p=$ $0.039)$. Women had lower SAD $(p=0.000)$ and GS $(p=0.045)$ compared to men. No difference between men and women was reported for BMl, systolic blood pressure, diastolic blood pressure, waist circumference and hip circumference.

The relationship between the GS and SAD categories in analyses of covariance is presented in Table 3 . The unadjusted means of GS were 0.995 (95\% Cl 0.972-1.019) m/s in SAD Q1 participants, 0.991 (95\% Cl 
0.968-1.014) $\mathrm{m} / \mathrm{s}$ in SAD Q2, $0.986(95 \% \mathrm{Cl} 0.964-1.007) \mathrm{m} / \mathrm{s}$ in SAD Q3 and 0.961 (95\% Cl 0.937-0.985) $\mathrm{m} / \mathrm{s}$ in SAD Q4 individuals in women. The similar trend presented in men [Q1: $0.993(95 \% \mathrm{Cl}$ 0.969-1.016) m/s; Q2: 0.980 (95\%Cl 0.956-1.004); Q3: 0.944 (95\%Cl 0.918-0.970); Q4: 0.948 (95\%Cl 0.923-0.973)]. After adjustment for age, the reported trends between GS and SAD in categories were largely confirmed in women, but not in men.

GS and SAD were dramatically significantly correlated $(r=-0.072, P=0.00)$. Linear trends between

SAD and GS were reported for women and men.

Table 3

Results from unadjusted and adjusted analyses of covariance presenting means (and 95\% confidenceintervals) of 4-meter gait speed according to sagittal abdominal diameter (SAD) categories, stratified by gender

\begin{tabular}{|lll|}
\hline & Age unadjusted 4m gait speed & Age adjusted 4m gait speed \\
\hline Women $(n=1554)$ & & \\
\hline Q1, SAD<18.0cm $(n=377)$ & $0.995(0.972 ; 1.019)$ & $0.976(0.954 ; 0.999)$ \\
\hline Q2, SAD18.0-19.79cm $(n=411)$ & $0.991(0.968 ; 1.014)$ & $0.961(0.940 ; 0.983)$ \\
\hline Q3, SAD19.8-21.89cm $(n=393)$ & $0.986(0.964 ; 1.007)$ & $0.936(0.915 ; 0.957)$ \\
\hline Q4, SAD $21.9 \mathrm{~cm}(n=373)$ & $0.961(0.937 ; 0.985)$ & $0.907(0.887 ; 0.928)$ \\
\hline P & 0.177 & 0.000 \\
\hline Men $(n=1291)$ & & $1.024(1.002 ; 1.047)$ \\
\hline Q1, SAD<18.0cm $(n=326)$ & $0.993(0.969 ; 1.016)$ & $1.008(0.986 ; 1.030)$ \\
\hline Q2, SAD18.0-19.79cm $(n=322)$ & $0.980(0.956 ; 1.004)$ & $1.005(0.981 ; 1.028)$ \\
\hline Q3, SAD19.8-21.89cm $(n=320)$ & $0.944(0.918 ; 0.970)$ & $1.023(0.998 ; 1.049)$ \\
\hline Q4, SAD $\geq 21.9 c m(n=323)$ & $0.948(0.923 ; 0.973)$ & 0.000 \\
\hline$P$ & 0.013 & \\
\hline
\end{tabular}

\section{Discussion}

In this cross-sectional analysis of persons aged 50 years and older, we found that GS follows a linear pattern across SAD categories. Compared to those in the lowest quartile of SAD $(<18 \mathrm{~cm})$, participants in the highest quartile $(>21.9 \mathrm{~cm})$ were more likely to have lower GS. These relationships are independent of age in women, but not in men.

$\mathrm{BMI}$ is utilized to confirm in case subjects are overweight or obese disregarding the distribution of body fat. High BMI is related with hypertension, diabetes mellitus and cardiovascular diseases. Abdominal 
adiposity is likewise a risk factor for the development of dyslipidemia, hypertension CVDs and glucose intolerance. Aggregating proof proposes that visceral adipose tissue is more emphatically identified with metabolic risk factors than subcutaneous adipose tissue. WC and SAD are utilized as abdominal obesity markers, yet WC doesn't recognize visceral from subcutaneous adipose tissue, and consequently can't precisely reflect levels of visceral adipose tissue. SAD gives a superior intra-abdominal or visceral obesity estimate than WC.

Our study revealed that the unadjusted means of GS decreased with increase of SAD. It presented a linear pattern both in men and in women and was confirmed by recent evidence. A few reports have shown that fatty infiltration of muscle is a significant part of low muscle strength and that abdominal obesity can lessen muscle strength through inflammatory and endocrine mechanisms [11-13]. Aging is associated with increased the inflammatory activity. Raised CRP levels have been related with incident mobility limitations in subjects with and without diabetes [14]. A new report found that WC was firmly associated with mobility disability and with activities of daily living disability and showed that obesity was associated with functional decline after some time [15].

We also found that the trend was largely confirmed in women, but not in men after adjustment for age. It is notable that ageing leads to reduction of functional ability and increase of visceral adipose tissue. Past studies have shown that body fat will redistribute and subcutaneous fat tissue will move to visceral adipose tissue with age [16]. The difference is partly explained by menopausal status. Several alterations in fat deposits occur with the advent of the menopause, leading to a change in the distribution of body fat. Postmenopausal women had a higher prevalence of central obesity [17].

The strengths of our study included that it provided the relationship between SAD and GS in a Chinese rural and village settings. However, there are some limitations in our study. First, the participants were recruited among persons aged 50 years and older living in rural areas. This may introduce a

representativeness bias for external validation of our results. Second, non-participants were older and had more hypertension which could lead to selection biases. Third, no conclusion in regard to the cause or consequence can be drawn, due to the cross-sectional approach.

\section{Conclusions}

A linear relationship between gait speed and SAD is here demonstrated. After age adjustment, the relationship exists in women, not in men. It may provide complementary information in the comprehensive assessment of older persons. Age and gender should be considered when we explore the relationship between physical function and visceral adipose tissue in elderly.

\section{Abbreviations}

SAD: Sagittal abdominal diameter; BMI: Body mass index; GS: Gait speed; Cl: Confidence interval;HES:Handan Eye Study; CVD: Cardiovascular disease; CRP: C-reactive protein 


\section{Declarations}

\section{Acknowledgement}

The authors acknowledgethe contribution of the study participants and the HESgroup involved in data collection.

\section{Authors' contributions}

JPL analyzed the data, participated in the manuscript writing and revision,finalized the results, and made the tables and figures. XRL and NLW providedcritical comments on the design and the results and conceived the studyideas. KC provided comments on data sources and the manuscript. JH and YZ involved data collection,managementand result interpretation. All authorsparticipated in the discussion and contributed to the final manuscript. Allauthors read and approved the submission of the final manuscript to BMCGeriatrics.

\section{Funding}

The projected is funded by a grant from the Ministry ofScience and Technology of China (No. 2007CB512201).

\section{Availability of data and materials}

Final analysis data will be available to the researchers upon request via email at

tydljp@126.com or by mail to Dr. Ningli Wang, Beijing Tongren Eye Center, Beijing TongrenHospital, Capital Medical University, Beijing Ophthalmology \& Visual Science KeyLaboratory,Beijing Institute of Ophthalmology, No.1. Dong Jiao Min Xiang,Dongcheng District,Beijing,100730, China.

\section{Ethics approval and consent to participate}

All the participants gave informed written consent, and the survey wasapproved by the Beijing Tongren Hospital Ethics Committee (TREC2006-22).

\section{Consent for publication}

Not applicable.

\section{Competing interests}

The authors declare no conflicts of interest in the present study.

\section{References}


1. Guralnik JM, Fried LPSalive ME Disability as a public health outcome in the aging population [J]. Annu Rev Public Health, 1996,17: 25-46. DOI: 10.1146/annurev.pu.17.050196.000325.

2. Cesari $M$, Onder $G$, Russo $A$, et al. Comorbidity and physical function: results from the aging and longevity study in the Sirente geographic area (ilSIRENTE study) [J]. Gerontology, 2006,52(1): 24-32. DOI: $10.1159 / 000089822$.

3. Firouzi SA, Tucker LA, LeCheminant JD, et al. Sagittal Abdominal Diameter, Waist Circumference, and BMI as Predictors of Multiple Measures of Glucose Metabolism: An NHANES Investigation of US Adults [J]. J Diabetes Res, 2018,2018: 3604108. DOI: 10.1155/2018/3604108.

4. Yim JY, Kim D, Lim SH, et al. Sagittal abdominal diameter is a strong anthropometric measure of visceral adipose tissue in the Asian general population [J]. Diabetes Care, 2010,33(12): 2665-2670. DOI: $10.2337 / \mathrm{dc} 10-0606$.

5. Chiles Shaffer N, Simonsick EM, Thorpe RJ, et al. The Roles of Body Composition and Specific Strength in the Relationship Between Race and Physical Performance in Older Adults [J]. J Gerontol A Biol Sci Med Sci, 2020,75(4): 784-791. DOI: 10.1093/gerona/glz103.

6. Tabue-Teguo M, Peres K, Simo N, et al. Gait speed and body mass index: Results from the AMI study [J]. PLoS One, 2020,15(3): e0229979. DOI: 10.1371/journal.pone.0229979.

7. Liang YB, Friedman DS, Wong TY, et al. Rationale, design, methodology, and baseline data of a population-based study in rural China: the Handan Eye Study [J]. Ophthalmic Epidemiol, 2009,16(2): 115-127. DOI: 10.1080/09286580902738159.

8. Cao K, Hao J, Zhang Y, et al. Design, methodology, and preliminary results of the follow-up of a population-based cohort study in rural area of northern China: Handan Eye Study [J]. Chin Med J (Engl), 2019,132(18): 2157-2167. DOI: 10.1097/CM9.0000000000000418.

9. Duarte Pimentel G, Portero-McLellan KC, Maesta N, et al. Accuracy of sagittal abdominal diameter as predictor of abdominal fat among Brazilian adults: a comparation with waist circumference [J]. Nutr Hosp, 2010,25(4): 656-661.

10. Abellan van Kan G, Rolland Y, Andrieu S, et al. Gait speed at usual pace as a predictor of adverse outcomes in community-dwelling older people an International Academy on Nutrition and Aging (IANA) Task Force [J]. J Nutr Health Aging, 2009,13(10): 881-889. DOI: 10.1007/s12603-009-0246-z.

11. Marcus RL, Addison O, Dibble LE, et al. Intramuscular adipose tissue, sarcopenia, and mobility function in older individuals [J]. J Aging Res, 2012,2012: 629637. DOI: 10.1155/2012/629637.

12. Goodpaster BH, Park SW, Harris TB, et al. The loss of skeletal muscle strength, mass, and quality in older adults: the health, aging and body composition study [J]. J Gerontol A Biol Sci Med Sci, 2006,61(10): 1059-1064. DOI: 10.1093/gerona/61.10.1059.

13. Delmonico MJ, Harris TB, Visser M, et al. Longitudinal study of muscle strength, quality, and adipose tissue infiltration [J]. Am J Clin Nutr, 2009,90(6): 1579-1585. DOI: 10.3945/ajcn.2009.28047.

14. Figaro MK, Kritchevsky SB, Resnick HE, et al. Diabetes, inflammation, and functional decline in older adults: findings from the Health, Aging and Body Composition (ABC) study [J]. Diabetes Care, 2006,29(9): 2039-2045. DOI: 10.2337/dc06-0245. 
15. Yang S, Wang S, Tai P, et al. Central and Peripheral Adiposity Had Different Effect on Disability in Centenarians [J]. Front Endocrinol (Lausanne), 2021,12: 635205. DOI: 10.3389/fendo.2021.635205.

16. Cartwright MJ, Tchkonia TKirkland JL Aging in adipocytes: potential impact of inherent, depotspecific mechanisms [J]. Exp Gerontol, 2007,42(6): 463-471. DOI: 10.1016/j.exger.2007.03.003.

17. Chen JL, Guo J, Mao P, et al. Are the factors associated with overweight/general obesity and abdominal obesity different depending on menopausal status? [J]. PLoS One, 2021,16(2): e0245150. DOI: $10.1371 /$ journal.pone.0245150.

18. Gravena AA, Brischiliari SC, Lopes TC, et al. Excess weight and abdominal obesity in postmenopausal Brazilian women: a population-based study [J]. BMC Womens Health, 2013,13: 46. DOI: $10.1186 / 1472-6874-13-46$. 